\title{
Lothar Gall
}

\section{Bismarcks Süddeutschlandpolitik 1866-1870}

In der Stagnation seiner Politik gegenüber Süddeutschland und den süddeutschen Staaten nach 1866/67 sei, so lautete viele Jahrzehnte die vorherrschende Meinung der Historiker, eines der wesentlichen Motive Bismarcks und der preußischen Politik zu suchen, einem militärischen Konflikt mit Frankreich nach 1867, nach Úberwindung der Luxemburg-Krise, zumindest nicht mehr auszuweichen. Das Ziel dieser Politik sei nun, nach 1866, darüber war man sich weithin einig, endgültig die Lösung der deutschen Frage im preußisch-kleindeutschen Sinne gewesen. Dieser Stagnationsthese hat zunächst Otto Becker ${ }^{1}$ und dann, auf einer wesentlich erweiterten Quellengrundlage, vor wenigen Jahren Rolf Wilhelm ${ }^{2}$ nachdrücklich widersprochen: Von einer Stagnation, gar von einem Scheitern könne keine Rede sein. Im Gegenteil. „Eine genaue Untersuchung des Verhältnisses der süddeutschen Staaten zum Norddeutschen Bund“ ergebe vielmehr, „daß in den Jahren vor dem deutsch-französischen Krieg eine Entwicklung im Gang war, die wenn Frankreich sie hinnahm - mit großer Wahrscheinlichkeit früher oder später zum Eintritt der süddeutschen Staaten in den Norddeutschen Bund führen mußte" . Anders gesagt: Bismarcks Politik gegenüber Süddeutschland und den süddeutschen Staaten sei in Anlage und Durchführung durchaus erfolgreich gewesen: „War der Krieg mit Frankreich unvermeidlich“, so Wilhelm, „so war er es also nicht, weil die Einigung mit dem Süden sonst an sich nicht hätte erreicht werden können, sondern weil Frankreich aus Gründen der traditionellen Gleichgewichtspolitik die Bildung einer hochgerüsteten Großmacht in der Mitte des Kontinents, die seit Bestehen des französischen Nationalstaats föderativ organisiert und offen für den französischen Einfluß war, glaubte verhindern zu müssen. “4

$\mathrm{Zu}$ einer vertieften Auseinandersetzung mit dieser Auffassung ist es allerdings bisher ebensowenig gekommen wie seinerzeit mit derjenigen Otto Beckers. Ähnliches gilt, und das ist noch sehr viel erstaunlicher, für eine Studie, die die Thesen-

${ }^{1}$ Bismarcks Ringen um Deutschlands Gestaltung. Hrsg. u. ergänzt von Alexander Scbarff (Heidelberg 1958).

${ }^{2}$ Das Verhāltnis der süddeutschen Staaten zum Norddeutschen Bund (1867-1870) (Husum 1978).

${ }^{3}$ Ebd. 163.

Ebd. $171 \mathrm{f}$. 
bildung sozusagen in die andere Richtung am weitesten vorangetrieben hat: für Josef Beckers Aufsatz „Zum Problem der Bismarckschen Politik in der spanischen Thronfrage 1870“, der im Juni 1971 in der „Historischen Zeitschrift“ erschien”. Obwohl hier, wenngleich in oft eher vorsichtigen Formulierungen, der Anteil Bismarcks und Preußens am Ausbruch des Krieges von 1870 sehr akzentuiert und gegenüber den bisher in der deutschen Geschichtswissenschaft vorherrschenden Einschätzungen wesentlich neu gewichtet wurde, löste der Beitrag, wenn ich richtig sehe, kaum Reaktionen aus, schon gar nicht solche, die etwa mit dem Echo auf Fritz Fischers Neuerörterung der Kriegsschuldfrage von 1914 zehn Jahre vorher vergleichbar gewesen wären.

Beckers Untersuchung, in deren Mittelpunkt die neuerliche akribische Rekonstruktion und Analyse der Vorgeschichte und Geschichte der spanischen Thronkandidatur stand, ging in wesentlichen Punkten ihrer Argumentation von der These aus, daß Bismarcks Süddeutschlandpolitik nach 1866 im Kern gescheitert sei und schließlich kaum noch Erfolgsaussichten im Sinne ihrer eigenen Zielsetzungen geboten habe. So habe sich eine Flucht nach vorne, das „Acheronta movebo“ eines neuen Krieges geradezu aufgedrāngt, eines „mit den Mitteln der klassischen Kabinettspolitik und im Geiste bonapartistischer Krisenbewältigung“ herbeigeführten „provozierten Defensivkrieges"6.

Erfolg, Stagnation, weitgehendes Scheitern - das umschreibt das Spektrum der Deutungen, die die Bismarcksche Süddeutschlandpolitik nach 1866, übrigens auch schon bei den Zeitgenossen, gefunden hat. Jede dieser Deutungen hat weitreichende Konsequenzen für die Interpretation der Bismarckschen Gesamtpolitik und insbesondere für seinen und Preußens Anteil an der Entstehung des Krieges von 1870: Es liegt auf der Hand, daß es hier stets zugleich eine Wechselwirkung gab und gibt, daß die Interpretation des Ausgangs sehr stark auch die Deutung der spezielleren Entwicklung, also die des Verhältnisses Preußens und des Norddeutschen Bundes zu Süddeutschland und den süddeutschen Staaten, bestimmt. Davon bei der Betrachtung und Analyse jener Entwicklung zu abstrahieren, ist kaum möglich und soll hier auch gar nicht versucht werden. Wohl aber erscheint es sinnvoll, jene Entwicklung etwas stärker, als dies bisher geschehen ist, unter dem Aspekt der möglichen Alternativen und der innen- und verfassungspolitischen Prioritäten zu betrachten.

Kaum etwas hat Perspektive und Einschätzung der Entwicklung nach 1866 und die Diskussion über sie nachhaltiger bestimmt als die These, es habe sich hier um eine durchaus provisorische Gestaltung der Verhāltnisse, um ein Provisorium gehandelt. Sicher, das entsprach der Auffassung vieler Zeitgenossen, wenngleich nicht so vieler und vor allem nicht mit so eindeutiger Zielrichtung, wie es dann im Zeichen der weiteren Entwicklung und der schließlich eindeutig vorherrschenden

\footnotetext{
${ }^{5} \mathrm{HZ} 212$ (1971) $529 \mathrm{ff}$.

${ }^{6}$ Ebd. 606.
} 
kleindeutsch-borussischen Interpretation dieser Entwicklung vielfach schien. Und sicher gab es auch eine Fülle von objektiven Elementen und Faktoren, die über die innen- und außenpolitische Situation, wie sie 1866 in Mitteleuropa entstanden war, hinausdrängten bzw. im Sinne der Wiederherstellung älterer Zustände, der Revision der „Ordnung von 1866 “, hinter sie zurück wollten. Blickt man jedoch speziell auf Bismarck und auf die innere, insbesondere auch verfassungspolitische Ordnung des Norddeutschen Bundes ${ }^{7}$, so wird man entgegen der bisher vielfach vorherrschenden Provisoriumsthese sagen können, daß bei dem, was der preußische Ministerpräsident hier der speziellen Konstellation des Jahres 1866, nicht zuletzt in Beschwörung nationaler Erwartungen, abgewann, die Elemente des Definitiven deutlich überwogen. Entscheidendes, so vor allem die Stellung des Monarchen und der von ihm allein bestimmten Exekutive, Rolle und Funktion des Parlaments einschließlich des so zentralen Budgetrechts, die Position von Heer und Bürokratie, aber auch die Grundprinzipien des Wirtschaftssystems und der sozialen Ordnung und des Verhältnisses zu den Kirchen wurden von nun an niemals mehr ernsthaft zur Diskussion gestellt. Hat man dies im Auge, so hatte Heinrich von Sybel mit seiner bewußt zuspitzenden Formulierung durchaus recht: „Im Herbste 1866 war das Deutsche Reich gegründet. ${ }^{\text {“8 }}$

Sybel hat daran - nicht als erster, aber mit besonders nachhaltiger Wirkung - die von Freund und Feind in der Folgezeit immer uneingeschränkter akzeptierte These geknüpft, die Nationalliberalen hätten sich im Interesse der nationalen Einheit rasch auf dieses Fundament gestellt und die darauf gegründete Politik, bei einigen Abweichungen im einzelnen und im Taktischen, im wesentlichen mitgetragen. Diese Politik sei die Politik des Anschlusses der süddeutschen Staaten an den neugegründeten Norddeutschen Bund gewesen, wie er, vor dem Hintergrund und unter den Bedingungen des Krieges gegen Frankreich im Spätherbst 1870, schließlich auch vollzogen worden sei.

Aus dieser Perspektive scheinen die Positionen und Fronten ganz klar: hier die Regierung Bismarck und die nationale Partei, sprich die Nationalliberalen und die ihr zuneigenden Kräfte im Süden Deutschlands, dort ihre gemeinsamen Gegner, eher schwach im Bereich des Norddeutschen Bundes, nach einer kurzen Zeit der Lähmung überraschend stark und an Zahl und Einfluß ständig zunehmend im Süden Deutschlands, mit der Habsburger Monarchie als ehemaliger Vormacht und heimlichem Protektor im Hintergrund. Und da, so kann man mit Heinrich Lutz die Linie ausziehen", von dieser Basis aus die Chancen für ein internationales Revi-

\footnotetext{
${ }^{7} \mathrm{Vgl}$. dazu jetzt ausführlich Klaus Erich Pollmann, Parlamentarismus im Norddeutschen Bund 1867-1870 (Düsseldorf 1985).

${ }^{8}$ Die Begründung des deutschen Reiches durch Wilhelm I., Bd.6 (München/Leipzig 1894) 21. Buch, 1. Kapitel.

${ }^{9}$ Heinricb Lutz, Österreich-Ungarn und die Gründung des Deutschen Reiches. Europāische Entscheidungen 1867-1871 (Frankfurt am Main/Berlin/Wien 1979).
} 
sionsbündnis zwischen den direkt oder indirekt Unterlegenen von 1866 zunächst nicht schlecht schienen, hatte die nationale Partei allen Anlaß, Bismarck keine Schwierigkeiten zu machen, sondern sich ihm in der Außenpolitik als Juniorpartner ganz zur Verfügung zu stellen.

Fraglos gab es eine Reihe von Krāften in der nationalliberalen Partei und Fraktion und bei den ihr Zuneigenden im Süden Deutschlands, die diese Einschätzung teilte und daraus die entsprechenden Konsequenzen zog: Die badische Regierung unter Karl Mathy und dann, nach Mathys Tod Anfang Februar 1868, unter Julius Jolly ist dafür in ihrer Spitze ein besonders handfestes Beispiel. Aber ebenso unbestreitbar ist - die innerparteiliche Reaktion auf die Politik der badischen Regierung macht das von der anderen Seite her gleichfalls besonders anschaulich -, daß diese Kräfte dabei teilweise auf sehr scharfen Widerstand in den eigenen Reihen stieBen ${ }^{10}$. Wurde dadurch doch das im Kern in Frage gestellt, was jenseits der vielbeschworenen „Kapitulation vor der Macht“ nicht nur nach außen zur Begründung der Annäherung an Bismarck und des teilweisen Ausgleichs mit ihm diente: daß die Einheit zur Freiheit führen werde und daß vor allem das konstitutionelle und liberale Erbe des deutschen Südens und Südwestens die Struktur des deutschen Nationalstaats entscheidend bestimmen, die im alten Preußen verkörperten obrigkeitsstaatlichen Elemente rasch in den Hintergrund drängen werde. Damit warben vor allem die süd- und südwestdeutschen Liberalen, die vielfach vor 1866 noch großdeutsch, zumindest aber antipreußisch und jedenfalls antibismarckisch gewesen waren, für die kleindeutsche Einigung. Und ihnen zerbröckelten die Argumente und mit ihnen die Anhängerschaft umso mehr, je deutlicher wurde, worauf die nationale Einigungspolitik, sprich die Politik des preußischen Ministerpräsidenten und neuen Kanzlers des Norddeutschen Bundes, gegenüber dem Süden Deutschlands hinauslief.

Diese Politik war, wie immer bei Bismarck, vielgleisig. Sie umfaßte Elemente der klassischen Allianzpolitik ebenso wie solche der Handels- und Zollpolitik, militärpolitische Abmachungen auf verschiedenen Ebenen ebenso wie Versuche, durch offene und versteckte Beeinflussung der Presse und durch die Schaffung eines speziellen „Zollparlaments“ Druck von unten zu erzeugen - Versuche, die wie im Fall der Zollparlamentswahlen vom Frühjahr 1868 auch einmal gründlich danebengehen konnten. Im Kern aber zielte sie, alle zentralen Dokumente sprechen hier bei unbefangener Prüfung eine sehr eindeutige Sprache, ganz klar darauf, die süddeutschen Monarchen und ihre Ratgeber und Regierungen davon zu überzeugen, daß ein kleindeutscher Nationalstaat unter preußischer - und das hieß zugleich unter seiner, Bismarcks - Führung innenpolitisch, was die inneren Machtverhältnisse an-

${ }^{10}$ Vgl. dazu Lothar Gall, Der Liberalismus als regierende Partei. Das Großherzogtum Baden zwischen Revolution und Reichsgründung (Wiesbaden 1968). 
ging, kein Instrument der Veränderung, sondern eines der Bewahrung sein werde $^{11}$

Der Tendenz dieser Politik entsprach es, daß Bismarck bei der Begründung des Norddeutschen Bundes auch und gerade nach außen die Tradition des Deutschen Bundes von 1815 sehr betonte. Und ebenso entsprach es ihr, daß der preußische Ministerpräsident die im Prager Frieden anvisierte Idee eines süddeutschen Bundes - der dann eines Tages vielleicht doch in irgendeiner Form, etwa in der eines „erweiterten Bundes“, mit dem Norddeutschen Bund würde verknüpft werden können - zu Zeiten wohl durchaus ernsthaft ventilierte. Vor allem aber fügt sich in diesen Zusammenhang, daß Bismarck die von Eduard Lasker Ende Februar 1870 im Reichstag des Norddeutschen Bundes vorgetragene Interpellation, wie die Regierung zu einem etwaigen Antrag Badens auf Aufnahme in den Bund stehe, mit ganz unerwarteter Schärfe beschied und dafür ohne weiteres eine schwere Belastung seines Verhältnisses zu den Nationalliberalen - und auch zur badischen Regierung - in Kauf nahm ${ }^{12}$ : Ein liberal, über Jahre sogar parlamentarisch regierter Staat, das konstitutionelle „Musterland“ Mitteleuropas, so demonstrierte er damit, könne keine besondere Behandlung erwarten, so entgegenkommend sich seine Regierung bis hinauf zu seinem mit dem preußischen Königshaus verschwāgerten Monarchen seit Jahren gegenüber Preußen und dem Norden verhalten habe. Im Gegenteil, die Richtung, die das Ganze dadurch angenommen habe, daß die Nationalliberalen versucht hätten, die Frage zu einer parlamentarischen Angelegenheit zu machen, sei ihm, Bismarck, durchaus zuwider: Initiativen und Entscheidungen auf diesem Gebiet seien allein Sache der Monarchen und der Regierungen, und er werde sich jedenfalls hinsichtlich des Verhältnisses zwischen Nord und Süd und der nationalen Frage nicht von liberalen Parteiüberlegungen leiten lassen.

Bismarcks Süddeutschlandpolitik nach 1866 stand also wie der gesamte Komplex seiner sogenannten nationalen Politik, so vielfältig und auch verwirrend seine Schachzüge im einzelnen oft waren, aufs Ganze gesehen eindeutig unter dem Primat machtpolitischer Strukturentscheidungen im Inneren, wie er sie nach $1866 \mathrm{im}$ Bereich des Norddeutschen Bundes durchgesetzt hatte und wie er sie um keinen Preis wieder zurücknehmen oder auch nur zur Diskussion stellen wollte, also auch nicht um den Preis rascherer Fortschritte in der Einigungsfrage. Für ihn war also der Norddeutsche Bund nicht, wie es dann die kleindeutsch-liberale Geschichtsschreibung immer wieder dargestellt hat, ein Provisorium, sondern in den entscheidenden Elementen seiner inneren Struktur etwas Definitives: eine äußere Erweiterung, so wünschenswert sie auch ihm aus nationalpolitischen und vor allem machtpolitischen Gründen schien, durfte seine innere Gestalt keineswegs in Frage stellen.

${ }^{11}$ So aus der Position der Gegnerschaft schon von den Zeitgenossen sehr klarsichtig: Josepb Edmund Jörg), Die neueste Improvisation des Grafen Bismarck, in: Historisch-politische Blätter für das katholische Deutschland 61 (1868) $637 \mathrm{ff}$.

12 Vgl. dazu Becker, Spanische Thronfrage (Anm. 5), $549 \mathrm{ff}$. 
Da sein Spielraum angesichts dessen einigermaßen eng war und, unerwartete äußere Anstöße und Ereignisse nicht einkalkuliert, eher enger wurde - mit ihrer Fortdauer überzeugten sich die streng monarchischen Kräfte in Bayern und Württemberg und auch die dortigen Monarchen zunehmend von der Haltbarkeit der neuen Verhältnisse -, stellte er sich selber und suchte er vor allem seine Umgebung und die ihm zugängliche Öffentlichkeit auf immer längere Fristen einzustellen. Leicht war das angesichts der Erwartungen, die das Jahr 1866 geweckt hatte, nicht und auch nicht ungefährlich: Zumal in der nationalliberalen Partei wuchs die Unruhe, und auf sie war er angesichts seines sich ständig verschlechternden Verhältnisses zu den Konservativen im Hinblick auf den Haushalt und die Gesetzgebung mehr als je zuvor angewiesen. Es kann jedoch keine Rede davon sein, daß er auch nur einen Augenblick erwogen habe, von seinem bisherigen Kurs in der Einigungsfrage abzuweichen. Er war und blieb offenkundig überzeugt, daß er die Sache erfolgreich werde durchstehen können.

Für die Nationalliberalen hingegen sah das Ganze sehr viel anders aus. Sie waren die nationale Partei schlechthin. Darauf, auf der Bündelung der Erwartungen, die sich auf den deutschen Nationalstaat in der jetzt praktisch nur noch denkbaren kleindeutschen Form richteten, beruhte ganz wesentlich ihre Anziehungskraft. Je mehr sich die Verhältnisse von der neuen Basis aus verfestigten, desto mehr war, zumal im Süden Deutschlands, diese Anziehungskraft bedroht: Die Zollparlamentswahlen von 1868 , aber auch die anschließenden Landtagswahlen zeigten das in sehr dramatischer Form. Dabei kam, wie gesagt, hinzu, daß ihr neuer Partner, der preußische Ministerpräsident und Kanzler des Norddeutschen Bundes, wenig dazu tat, die innenpolitischen Erwartungen zu nähren, die breitere Volksschichten mit der nationalen Einigung verbanden. Im Gegenteil. Mit dem preußischen Heeressystem, das die süddeutschen Staaten in mehr oder weniger modifizierter Form nach 1866 einführten, mit den Kosten, die es verursachte, mit der Verfassung des Norddeutschen Bundes und ihrer Praxis, mit den außen- und handelspolitischen Úberlegenheitsgebärden der Vor- und Großmacht zeigten sich Preußen und sein Regierungschef immer wieder von einer eher unvorteilhaften Seite. Die Warnungen der politischen Gegner vor einer „Verpreußung“ des Südens, vor einem Verlust der eigenen Freiheiten, der eigenen Lebensart, der eigenen Traditionen, vor dem Aufgehen in einem autoritären „Kasernenstaat“, fanden hier in der Wirklichkeit stāndig neue Nahrung.

„Ein einiges Deutschland haben wir gewollt, aber kein durch Annexion vergrößertes Preußen. Und das ist der Inhalt und der Ausgang der norddeutschen Bundesverfassung “, erklärte selbst ein eher auf dem rechten Flügel der Liberalen angesiedelter Mann wie der damalige württembergische Justizminister und spätere Ministerpräsident Hermann von Mittnacht 1867 in einer Wahlrede ${ }^{13}$. Angesichts des-

${ }^{13}$ Walter Seefried, Mittnacht und die deutsche Frage (Stuttgart 1928) 71. Weit schärfer hieß es in dem in Mannheim erscheinenden „Organ der deutschen Volkspartei“, dem „Deutschen Wochenblatt“, also dem Sprachrohr der süddeutschen Demokraten, der Entwurf für eine 
sen begannen sich in der nationalen Partei die Hoffnungen mehr und mehr auf ein neuerliches großes und massenbewegendes, die Szenerie von Grund auf veränderndes außenpolitisches Ereignis, auf einen einheitsstiftenden Konflikt zu richten. Eduard Lasker, der immer wieder betonte, die Verfassung des Norddeutschen Bundes müsse nach der Aufnahme der süddeutschen Staaten gründlich revidiert wer$\operatorname{den}^{14}$, soll in dieser Beziehung schon 1868 in seinem sächsischen Wahlkreis erstaunliche Reden gehalten haben ${ }^{15}$, und jedenfalls wuchs seit 1869 im kleindeutsch-nationalen Lager die Zahl der Stimmen beträchtlich an, die auf ein entschlossenes Vorgehen in der Einigungsfrage, ja auf einen Konfrontationskurs gegenüber Frankreich drängten ${ }^{16}$ : Das Kaiserreich sollte klar vor die Alternative gestellt werden, die kleindeutsche Einigung hinzunehmen oder einen Nationalkrieg zu riskieren.

Mehr als Reden und Zeitungsartikel standen freilich der nationalen Partei nicht zu Gebote, und seit Schleswig-Holstein glaubte Bismarck genau zu wissen, was von der Kraft und Selbstāndigkeit solcher „publizistischer“ Bewegungen zu halten sei. Immerhin aber hielt er es, neben den schon genannten Motiven, doch für geboten, anläßlich der Baden-Interpellation Laskers die Grenzen liberaler Aktivitäten und Initiativen auf dem Gebiet der auswärtigen Politik noch einmal ganz scharf zu markieren. Ob er selber die Dinge vielleicht im Kern ähnlich sah, spielte dabei keine Rolle. Er wāre wohl im Gegenteil eher geneigt gewesen, etwaige eigene Pläne in dieser Richtung zurückzustellen, als auch nur den Anschein zu erwecken, der Wunsch nach Fortschritten in der Einigungsfrage sei möglicherweise ein Nasenring, an dem man ihn schließlich doch in eine Richtung führen könne, in die er nicht wollte.

Von einer Partnerschaft mit taktischen Divergenzen zwischen Bismarck und den Nationalliberalen kann also bei genauerem Zusehen auch und gerade hinsichtlich der Einigungsfrage nach 1866 in keiner Weise die Rede sein. Die Erwartungen und Zielsetzungen beider Seiten klafften vielmehr immer weiter auseinander. „Auf das große Programm Bismarcks in der süddeutschen Frage“, schrieb Hermann Baumgarten Anfang Mārz 1870 an Heinrich v. Treitschke, ,gebe ich sehr wenig. Es ist vor der Hand nichts als eine Phantasie, eine ferne Möglichkeit, der er selber nach Kräften die realen Bedingungen raubt. Denn was wir heute in Bayern und

Verfassung des Norddeutschen Bundes sei der „Versuch, ein Militärgesetz zur Verfassung zu erheben, die nationalen Wünsche und Bestrebungen des Volkes mit der gleichförmigen Uniformierung, gleichen Bewaffnung und gleichen Dressur abzuspeisen und den Drang des Volkes für freiheitliche Entwicklung zur Stärkung der Macht des Staates, zur Stütze des Königtums in seinen willkürlichen Ausschreitungen zu mißbrauchen": 23. Februar 1869, in: KarlGeorg Faber, Die nationalpolitische Publizistik Deutschlands 1866-1871. Eine kritische Bibliographie, Bd. 1 (Düsseldorf 1961) 219.

${ }^{14}$ Vgl. etwa seine Rede vom 28.4. 1867: Faber, Nationalpolitische Publizistik (Anm. 13), 208.

15 Becker, Spanische Thronfrage (Anm. 5), $550 \mathrm{f}$.

${ }^{16}$ An ihrer Spitze stand bereits seit 1867 die in Leipzig von Gustav Freytag (seit 1869 gemeinsam mit Julius Eckardt) herausgegebene Zeitschrift „Die Grenzboten“. 
Württemberg sehen, das ist wesentlich die Frucht dieser grundverkehrten Politik, die Feinde zu schonen und die Freunde mit Füßen zu treten. Dieser Politik hat er am 24. Februar" - dem Tag seiner Antwort auf die Interpellation Laskers - „die Krone aufgesetzt." Und fünf Tage später: „Bismarck weiß vom Süden nichts und seine sehr mittelmäßigen Gesandten nicht mehr. Außerdem aber - und das ist die Hauptsache - steht das Fundament seiner Weltanschauung dem entgegen, was unser Volk wollen muß.“17

Bismarck gab sich über diese Stimmung und Einschätzung keinen Illusionen hin. Er wußte in jeder Phase nur zu gut - eine Fülle von Äußerungen bei unterschiedlichster Gelegenheit machte das sehr deutlich -, daß die Mehrheit der Nationalliberalen mit der Einigung und Einheit Hoffnungen verband, die mit dem, was er politisch, allgemein und für sich persönlich anstrebte, letztlich unvereinbar waren. In diesem Sinne war die Einigung hier wie dort kein Wert an sich, für den man letztlich fast jeden Preis zu zahlen bereit gewesen wäre, sondern in beiden Fällen ein Mittel zu unterschiedlichen Zwecken und Zielen, ein Faktor in einer Kosten-Nutzen-Rechnung, die jeweils sehr verschieden aussah.

Ist man an diesem Punkt angelangt, so ordnen sich die Fakten und Problemzusammenhänge im Verhältnis zwischen Norddeutschem Bund und Süddeutschland nach einem sehr klaren Muster. Von einem Versuch des wirklichen Werbens Bismarcks um Süddeutschland, um seine Bevölkerung und seine einzelnen Parteien kann danach definitiv keine Rede mehr sein. Es ging ihm zu keinem Zeitpunkt um die Entfesselung einer Anschlußbewegung: Sie würde, darüber war er sich vollständig im klaren, und die Mehrheit der Nationalliberalen ließ darüber auch keinen Zweifel aufkommen, im Falle des Erfolges sogleich verstärkte politische Ansprüche geltend machen. Worum es ihm ging, war, gleichsam objektive Bindungen und strukturelle Einflußmöglichkeiten zu schaffen, im Heerwesen, in der Handels- und Zollpolitik, durch Allianzautomatismen, durch Angleichung gesetzlicher Normen auf vielen Gebieten. Es sollte so ein immer dichter werdendes Geflecht entstehen, das schließlich die Monarchen und ihre Regierungen auch in dieser Hinsicht aus Gründen der Staats- und Machtrāson zum Anschluß an den Norden veranlassen würde.

Charakteristisch dafür ist, daß Bismarck auch hier Ende der sechziger Jahre den Hauptansatzpunkt, Druck auszuüben, wieder, wie zu Beginn seiner Amtszeit, in den Zollvereinsverträgen und ihrer Verlängerung sah: Sie bildeten die Interessenklammer, die Nord und Süd seit Jahrzehnten verband und deren Existenz selbst im Lager der eingefleischtesten „Partikularisten“ nur von ganz wenigen in Frage gestellt wurde ${ }^{18}$. Alle seine Mahnungen zur Geduld, seine Warnungen vor dem

17 7. bzw. 12. Mărz 1870: Deutscher Liberalismus im Zeitalter Bismarcks. Eine politische Briefsammlung, Bd. 1. Hrsg. v. Julius Heyderboff (Bonn/Leipzig 1925) 464 bzw. 466.

${ }^{18}$ Ein Beispiel für eine solche grundsätzlich argumentierende Kritik am Zollverein bei Bekker, Spanische Thronfrage (Anm.5), 522, Anm.52, der freilich dessen Tragweite m.E. etwas überschätzt. 
„Abschlagen unreifer Früchte“19 muß man vor diesem Hintergrund sehen. Sie beruhten auf der entschiedenen Ablehnung des Gedankens, daß der deutsche Nationalstaat schließlich doch noch aus einem Akt kollektiver Selbstbestimmung hervorgehen könnte, mit all den politischen Konsequenzen, die das zu haben drohte.

Denn auch wenn sich die gegenteilige Meinung tief eingewurzelt hat: Was die Nationalliberalen um Eduard Lasker als Konzeption entwickelten, bildete durchaus eine Alternative. Und niemand kann im nachhinein sagen, wie sich die französische Seite entschieden hätte, wenn sie vor die Wahl gestellt worden wäre, sich dem parlamentarisch abgesicherten und legitimierten Anschluß Badens mitsamt allem, was davon an Impulsen ausgehen konnte, zu beugen oder einen Krieg auszulösen. Daß andererseits eine Kriegserklärung wegen Baden und seiner Anschlußentscheidung auf deutscher, sprich süddeutscher Seite nicht die gleichen Konsequenzen gehabt hātte wie die Vorgänge im Juli im Zusammenhang mit der spanischen Thronkandidatur, ist angesichts der Haltung auch der großdeutsch-katholischen und „partikularistischen“ Kräfte gegenüber Frankreich ${ }^{20}$ nur schwer zu glauben. Eines aber wäre jedenfalls anders gewesen, und das war für Bismarck entscheidend: Es wäre vom ersten Tag an ein Krieg um die deutsche Einheit gewesen mit einer ganz anderen Stellung derjenigen, die mit einem deutschen Nationalstaat durchaus andere Vorstellungen verbanden als die eines Großpreußen bzw. diejenige eines Schutzverbandes zur Aufrechterhaltung des "monarchischen Prinzips“ nach Art eines weiterentwickelten Deutschen Bundes. Mit einem Wort: Das Ganze drohte dann auch innenpolitisch zu einem Nationalkrieg zu werden, der sich leicht der Steuerung und Beherrschung entzog, auf die es Bismarck so sehr ankam und die ihm im Herbst und Winter 1870/71 unter für ihn ungleich günstigeren Bedingungen dann so meisterhaft gelang.

Hier mündet die Frage nach Bismarcks Süddeutschlandpolitik, sprich nach seiner deutschen Politik nach 1866, unvermeidlicherweise wieder ein in die Frage nach seinem Anteil an dem Ausbruch des Krieges von 1870. Sie ist hier nicht mein Thema. Ich habe an anderer Stelle meine Meinung dargelegt, daß er ihn weder bewußt und bis in die letzten Einzelheiten inszeniert habe noch aber auch ihm ausgewichen $\operatorname{sei}^{21}$. Aus dem hier Dargelegten ergibt sich zusätzlich, daß ihm, so wie sich die Dinge und vor allem auch sein Verhältnis zu der nationalen Partei, zu den Nationalliberalen entwickelt hatte, die Art und die Bedingungen, unter denen

19 20. Februar 1869, Erlaß an den Münchener Gesandten Werthern, Gesammelte Werke (Friedrichsruher Ausgabe) VI b, 2.

${ }^{20} \mathrm{Vgl}$. Hugo Lacher, Politischer Katholizismus und kleindeutsche Reichsgründung. Eine Studie zur politischen Ideenwelt im deutschen Katholizismus, 1859-1871 (Diss. Mainz 1963) 207 ff.; s. a. Rudolf Lill, Die deutschen Katholiken und Bismarcks Reichsgründung, in: Theodor Schieder/Ernst Deuerlein (Hrsg.), Reichsgründung 1870/71 (Stuttgart 1970) 345 ff., und der Beitrag v. Heinrich Lutz in diesem Band.

${ }^{21}$ Bismarck. Der weiße Revolutionär (Frankfurt am Main/Berlin/Wien '1981) $414 \mathrm{ff}$. 
der Krieg sich ihm darbot, jedenfalls hochwillkommen waren. Sie erst erlaubten ihm die weitgehende Kontrolle des Ablaufs der Entwicklung und neben der Beherrschung der außenpolitischen auch die der innenpolitischen Risiken. So brauchte er schließlich, was die innere Gestalt des neugegründeten Reiches betraf, keine substantiellen Kompromisse einzugehen: Es blieb im wesentlichen beim bloßen Anschluß der süddeutschen Staaten an den Bund, so wie er ihn im Herbst und Winter 1866/67, auf einem Höhepunkt seiner Macht und seines politischen Einflusses, ins Leben gerufen hatte.

Daß ihm bei diesem neuerlichen Erfolg nicht nur das Glück, die Gunst der Umstände entgegengekommen seien - dieser Gedanke liegt nahe. Beweisen aber lassen sich, wie mir scheint, die darauf gegründeten Hypothesen bis heute nicht, und auch die Geschichte seiner Politik gegenüber den süddeutschen Staaten in den Jahren 1866 bis 1870 präsentiert sich bei nüchterner Betrachtung wohl doch nicht so, daß man daraus einen $Z$ wang zu einer Flucht nach vorne ableiten müßte. 